\title{
V23. RECOMBINANT PROTEINS OF HPVI 6 CAPSID EXPRESSED IN 293-F CELLS CULTURED IN SUSPENSION AND SERUM FREE MEDIUM FOR VACCINE DEVELOPMENT.
}

Dirce Sakauchi ${ }^{1}$; Érica Akemi Kavati ${ }^{1}$; Fernanda de Oliveira Bou Anni ${ }^{1}$; Balasubramanyam Karanam²; Richard Roden³; Martin Müller4; Aurora Marques Cianciarullo ${ }^{1}$.

${ }^{1}$ Laboratory of Genetics, Butantan Institute, Dr. Vital Brasil, Brazil;

${ }^{2}$ Department of Biology and Cancer Research, USA;

${ }^{3}$ Department of Pathology, Johns Hopkins University, USA;

${ }^{4}$ Tumorvirus-specific Vaccination Strategies, Infection, Inflammation and Cancer Program, German Cancer Research Center (DKFZ), Germany.

INTRODUCTION Cervical cancer is the most serious consequence of infection by human papillomavirus (HPV), constituting one of the leading causes of death among women worldwide, and a fact that points to a major challenge to global public health. The prophylactic vaccination on a large scale would be an alternative to reducing cervical cancer rates, making it essential to search for new vaccine strategies. Current vaccines are based on virus-like particles (VLPs) of the major virus capsid protein L1, with effective protection against specific types, but with a limited cross-protection against other HPV types. The vaccine proposal is to use the L2 protein, which contains highly conserved sequences for a broader protection against different types of HPVs.

OBJECTIVE In this study, we have developed the production of VLPs containing L1 and L2 recombinant proteins of HPV16, using human embryonic kidney cells (293-F) suspended in medium fetal bovine serum free.

METHODOLOGY Cells 293-F were cultivated in serum-free medium and cotransfected with expression vectors for mammals pUF3L1 (L1) and pU3L2h (L2). The expression of the proteins L1 and L2 were analyzed by confocal laser microscopy, flow cytometry and transmission electron microscopy. The recombinant proteins HPV16L1L2 were purifiedby affinity chromatography with heparin resin and immunized in Balb/c mice subcutaneously with three doses of HPV16L1L2 formulated with aluminum hydroxide as an adjuvant and used saline solution as control. The immune response induced by recombinant HPV16L1L2 was evaluated by Elisa test and western-blotting. 
RESULTS The kinetics of expression of the HPV16 proteins L1L2 showed that maximal expression occurs around $48 \mathrm{~h}$ post-transfection by confocal microscopy and flow cytometry. Both proteins were localized in the nucleus and cytoplasm of cells. The analysis by transmission electron microscopy showed the formation of VLPs and the presence of proteins L1 and L2 through colloidal gold immunolabeling, in cell lysate. Purification by affinity chromatography allows obtaining VLPs more structurally correct. The immunoassays by Western blotting and ELISA confirmed the induction of immune response to both proteins by the immune system of mice. The serum antiHPV16 L1 and anti-HPV16 L2 of animals immunized with VLPs L1L2 recognized the major protein $\mathrm{L} 1$ molecular weight around $55 \mathrm{kDa}$ and the secondary protein $\mathrm{L} 2$ of approximately $72 \mathrm{kDa}$, respectively. The ELISA assays demonstrated that serum antibodies showed anti-HPV16 L1 titers above in relation to anti-HPV16 L2 in the group immunized with adjuvant.

CONCLUSION New strategies should be experienced to optimize the immunogenicity of VLPs L1L2 of HPV16. However, VLPs L1L2 of HPV16 production system was established efficiently in human epithelial cells in suspension and with promising results, which may contribute to the development of a prophylactic vaccine with broad protection spectrum against diverse HPV types.

KEYWORDS human papillomavirus, cancer, HPV16, VLP, L1L2, prophylactic vaccine. 